\title{
ON THE AVERAGE ORDER OF SOME ARITHMETICAL FUNCTIONS
}

\author{
BY BRUCE C. BERNDT ${ }^{1}$
}

Communicated by Paul T. Bateman, February 3, 1970

\begin{abstract}
We consider a large class of arithmetical functions generated by Dirichlet series satisfying a functional equation with gamma factors. Our objective is to state some $\Omega$ results for the average order of these arithmetical functions.
\end{abstract}

Our objective here is to state some $\Omega$-theorems on the average order of a class of arithmetical functions.

We indicate very briefly the class of arithmetical functions under consideration. For a more complete description, see [4].

Let $\{a(n)\}$ and $\{b(n)\}$ be two sequences of complex numbers, not identically zero. Let $\left\{\lambda_{n}\right\}$ and $\left\{\mu_{n}\right\}$ be two strictly increasing sequences of positive numbers tending to $\infty$. Put $s=\sigma+i t$ with $\sigma$ and $t$ both real and suppose that

$$
\phi(s)=\sum_{n=1}^{\infty} a(n) \lambda_{n}^{-s} \text { and } \psi(s)=\sum_{n=1}^{\infty} b(n) \mu_{n}^{-s}
$$

each converge in some half-plane. Let $\sigma_{a}^{*}$ denote the abscissa of absolute convergence of $\psi$. Put

$$
\Delta(s)=\prod_{\nu=1}^{N} \Gamma\left(\alpha_{v} s+\beta_{v}\right)
$$

where $\alpha_{\nu}>0$ and $\beta_{\nu}$ is complex, $\nu=1, \cdots, N$. Assume that for sone real number $r, \phi$ and $\psi$ satisfy the functional equation $\Delta(s) \phi(s)$ $=\Delta(r-s) \psi(r-s)$.

We shall consider the Riesz sum

$$
A_{q}(x)=\frac{1}{\Gamma(q+1)} \sum_{\lambda_{n} \leq x} a(n)\left(x-\lambda_{n}\right)^{q},
$$

where $q \geqq 0$. Let $\alpha=\sum_{v=1}^{N} \alpha_{\nu}$ and define

$$
Q_{q}(x)=\frac{1}{2 \pi i} \int \frac{\Gamma(s) \phi(s) x^{s+q}}{c_{q} \Gamma(s+q+1)} d s,
$$

AMS Subject Classifications. Primary 1043; Secondary 1040, 1041.

Key Words and Phrases. Arithmetical function, functional equation with gamma factors, Dirichlet series, average order.

1 Research partially supported by NSF Grant \# GP-7506. 
where $C_{q}$ is a cycle encircling all of the singularities of the integrand to the right of $\sigma=-q-1-k$, where $k>\left|\frac{1}{2} r-1 /(4 \alpha)\right|$, and where all of the singularities of $\phi$ lie in $\sigma>-k$. Then, the "error term" $P_{q}(x)$ is defined by

$$
P_{q}(x)=A_{q}(x)-Q_{q}(x) .
$$

Furthermore, let

$$
\begin{aligned}
& \beta(q)=\beta=-\sum_{\nu=1}^{N} \beta_{\nu}+\frac{1}{2} N-\frac{1}{2} r \alpha-\frac{3}{4}-\frac{1}{2} q, \\
& \theta(q)=\theta=\frac{1}{2} r-1 /(4 \alpha)+q-q /(2 \alpha),
\end{aligned}
$$

and

$$
\kappa(q)=\kappa=\sigma_{a}^{*}-\frac{1}{2} r-1 /(4 \alpha)-q /(2 \alpha) .
$$

From [4, p. 111], $\kappa(0) \geqq 0$. In the sequel we assume that $\kappa(q) \geqq 0$. If $\kappa(q)<0$, the order of $P_{q}(x)$ can be determined exactly [4, Theorem 3.2].

We are now ready to state

TheOREM 1. Assume that $b(n) \geqq 0$ and that $\beta_{\nu}$ is real, $\nu=1, \cdots, N$. Suppose that there exist constants $c$ and $\rho$ such that as $x$ tends to $\infty$,

$$
\sum_{\mu_{n} \leq x} b(n) \sim c x^{*} \log ^{\rho-1} x .
$$

Lastly, suppose that $\mu_{n+1}-\mu_{n}=o\left(\mu_{n}\right)$, as $n$ tends to $\infty$. Then, if $\cos (\beta \pi)$ $>0$ and $\kappa>0$,

$$
\operatorname{Re}\left\{P_{q}(x)\right\}=\Omega_{+}\left(x^{\theta}\{\log x\}^{\times}\{\log \log x\}^{p-1}\right) ;
$$

if $\cos (\beta \pi)<0$ and $\kappa>0$,

$$
\operatorname{Re}\left\{P_{q}(x)\right\}=\Omega_{-}\left(x^{\theta}\{\log x\} \times\{\log \log x\}^{\rho-1}\right) .
$$

The proof of Theorem 1 for $q=0$ is given in [1]. The proof of the more general theorem given here follows along the same lines. The idea of the proof goes back to Szegö [7] and Szegö and Walfisz [8]. Dirichlet's theorem on the simultaneous approximation of a finite set of real numbers is used in the proof, and it is at this stage of the proof that the restriction $b(n) \geqq 0$ is necessary.

Results of Hardy [5] on $r_{2}(n)$, the number of representations of $n$ as the sum of two squares, and on $d(n)$, the divisor function, are special cases of Theorem 1. Results of Szegö [7] on $r_{k}(n)$ and Szegö and Walfisz [8] on the Piltz divisor problem in algebraic number fields are also special cases.

For the arithmetical functions under consideration, Theorem 1 is an 
improvement upon general theorems of Landau [6] and Chandrasekharan and Narasimhan [3], [4].

Theorem 1 yields only "one-sided" results. In many cases, however, we can obtain "two-sided" results as the following theorem shows.

Theorem 2. Assume the hypotheses of Theorem 1. Let $Q_{\psi}(x)$ be $Q_{0}(x)$ except that $\phi$ is replaced by $\psi$. Suppose that as $x$ tends to $\infty$,

$$
Q_{\psi}^{\prime}(x) \sim c \sigma_{a}^{*} x^{\sigma_{a}^{*}-1} \log ^{\rho-1} x .
$$

Let $\gamma(q)=\gamma=2 \alpha \kappa-1$, and for $\kappa>0$ and a real define

$$
g(a)=\int_{0}^{\infty} e^{-u^{2}} u^{\gamma} \cos (a u+\beta \pi) d u .
$$

Then, if $\kappa>0$ and $g(a)$ has a change in sign,

$$
\operatorname{Re}\left\{P_{q}(x)\right\}=\Omega_{ \pm}\left(x^{\theta}\{\log x\} \times\{\log \log x\}^{\rho-1}\right) ;
$$

if $\kappa=0$, in all cases,

$$
\operatorname{Re}\left\{P_{q}(x)\right\}=\Omega_{ \pm}\left(x^{\theta}\{\log \log x\}^{p}\right) .
$$

The assumption in Theorem 1 that $\cos (\beta \pi) \neq 0$ has been removed. However, we have an additional restriction in that $g(a)$ has a change in sign. In [2] we establish some general conditions under which $g(a)$ has a sign change. We also determine there some conditions under which $g(a)$ has no sign change. It is very unfortunate, indeed, that the most interesting cases of $r_{2}(n)$ and $d(n)$ for $q=0$ fall into this latter category.

The proof of Theorem 2 for $q=0$ and $\kappa>0$ is given in [2], and the proof for $q>0$ follows along the same lines. For $\kappa=0$, the proof is, in fact, somewhat easier. The idea for the proof of Theorem 2 goes back to Szegö and Walfisz [9], and so their results on the Piltz divisor problem for algebraic number fields are special cases of Theorem 2. Again, Dirichlet's theorem is used in the proof, but in a different way, however.

Our next theorem yields some information on how of ten the inequalities (1) and (2) in Theorem 2 are valid.

Theorem 3. Assume the hypotheses of Theorem 2. Then, there exist positive constants $c_{1}$ and $c_{2}$ and a positive, strictly increasing sequence $\left\{y_{n}\right\}$ tending to $\infty$ such that both inequalities

$$
\pm \operatorname{Re}\left\{P_{q}(x)\right\}>c_{1} x^{\theta}(\log x)^{\times}(\log \log x)^{\rho-1}
$$


if $\kappa>0$, and

$$
\pm \operatorname{Re}\left\{P_{Q}(x)\right\}>c_{1} x^{\theta}(\log \log x)^{\rho}
$$

if $\kappa=0$, have solutions in each interval

$$
y_{n} \leqq x \leqq y_{n}+c_{2} y_{n}^{1-1 /(2 \alpha)}\left(\log y_{n}\right)^{1 / 2-1 /(2 \alpha)} .
$$

For $\kappa>0$ and $q=0$ the proof is given in [2]. The proof of the more general Theorem 3 is exactly the same. Theorem 3 gives an improvement upon a general theorem of Landau [6] for the arithmetical functions under consideration.

The author is grateful to John Steinig for several critical comments concerning [1], [2] and this paper.

\section{REFERENCES}

1. Bruce C. Berndt, On the average order of a class of arithmetical functions. I, J. Number Theory (to appear).

2. - On the average order of a class of arithmetical functions. II, J. Number Theory (to appear).

3. $\mathrm{K}$. Chandrasekharan and Raghavan Narasimhan, Hecke's functional equation and the average order of arithmetical functions, Acta Arith. 6 (1960/61), 487-503. MR 23 \#A3719.

4. - Functional equations with multiple gamma factors and the average order of arithmetical functions, Ann. of Math. (2) 76 (1962), 93-136. MR 25 \#3911.

5. G. H. Hardy, On Dirichlet's divisor problem, Proc. London Math. Soc. (2) 15 (1916), 1-25.

6. E. Landau, Über die Anzahl der Gitterpunkte in gewissen Bereichen, Abh. Nachr. Ges. Wiss. Göttingen 4 (1924), 137-150.

7. G. Szegö, Beiträge zur Theorie der Laguerreschen Polynome. II: Zahlentheoretische Anwendungen, Math. Z. 25 (1926), 388-404.

8. G. Szegö und A. Walfisz, Über das Piltzsche Teilerproblem in algebraischen Zahlkörpern. I, Math. Z. 26 (1927), 138-156.

9. - Über das Piltzsche Teilerproblem in algebraischen Zahlkörpern. II, Math. Z. 26 (1927), 467-486.

University OF IllinoIs, Urbana, IllinoIs 61801 